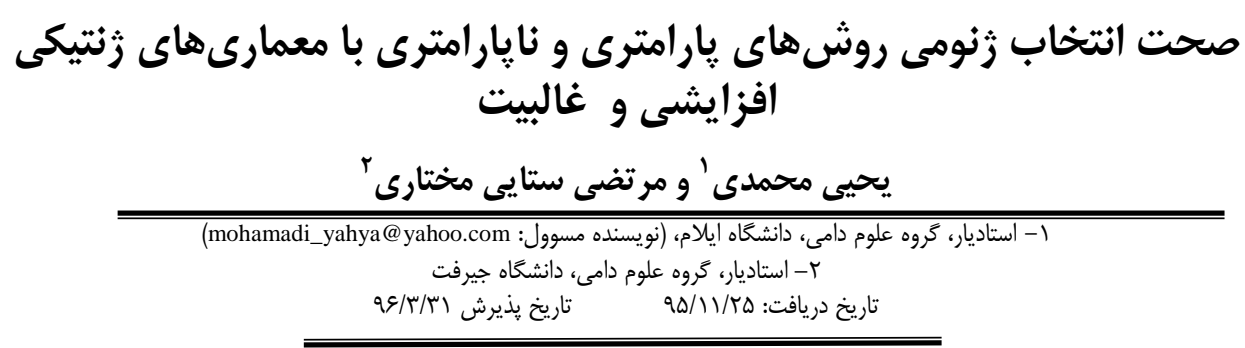

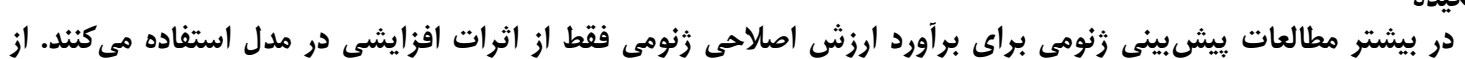

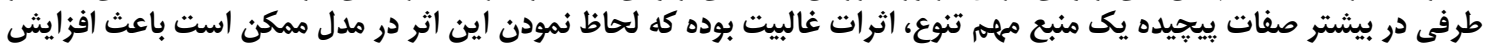

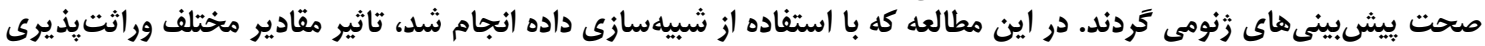

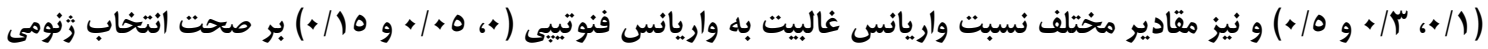

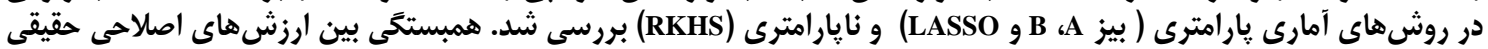

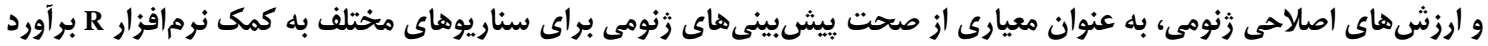

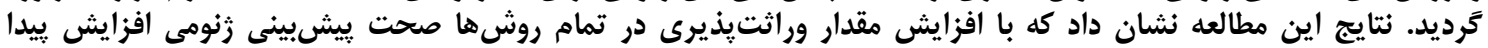

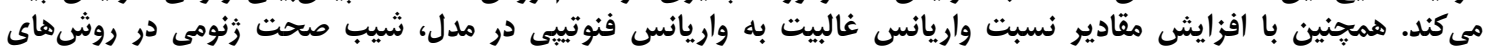

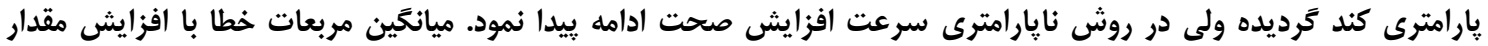

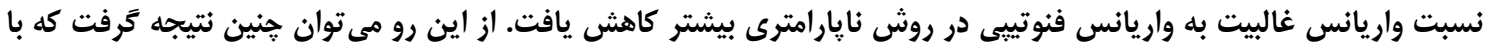

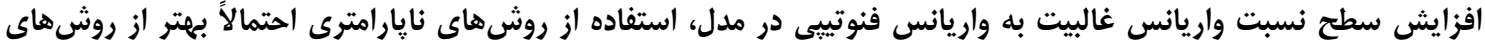
يارامترى صحت بيشبينى نهاى زنومى را برآورد مى كنند.

وازههاى كليدى: اثر افزايشى، اثر غالبيت، انتخاب زنومى، صحت انتخاب، شبيهسازى

استفاده از روش انتخاب زنومى، مدلها و روشهاى آمارى

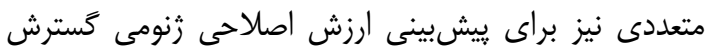

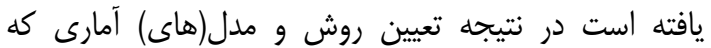

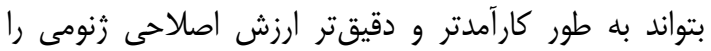

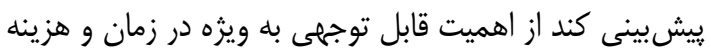

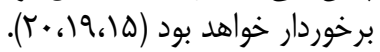

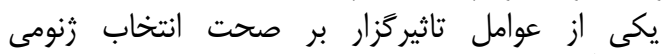

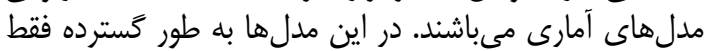

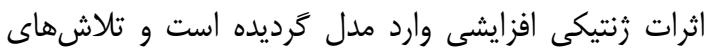

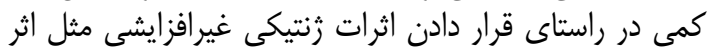

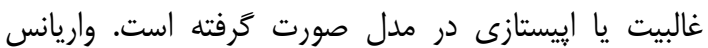

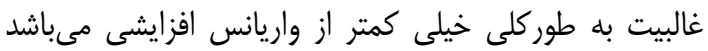

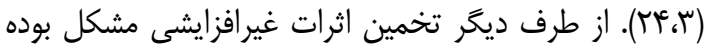

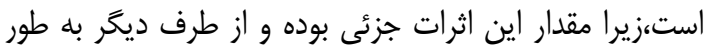

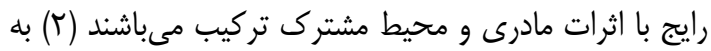

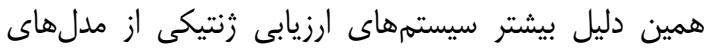

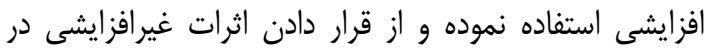

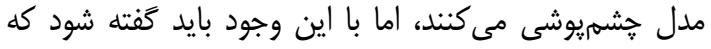

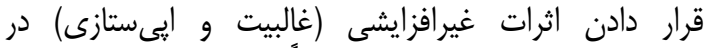

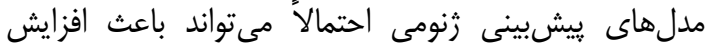
صحت گردد.

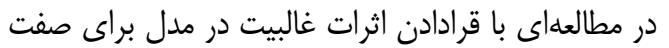

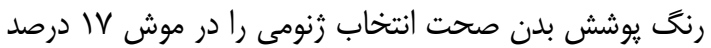

مقدمه

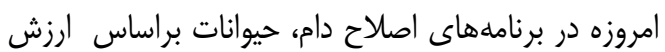

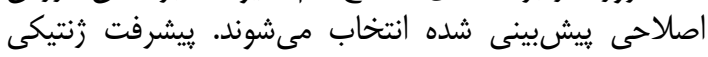

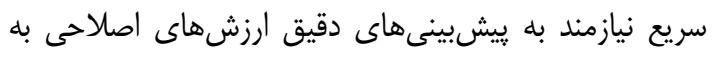

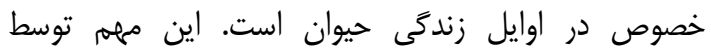

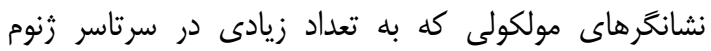

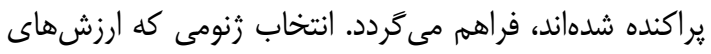

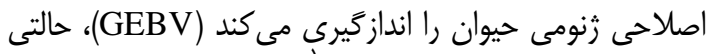

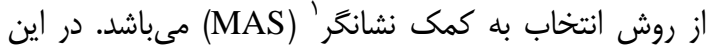

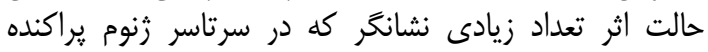

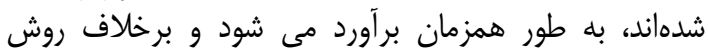
كه درصد كمى از واريانس زنتيكى صفت من رارد را توجيه

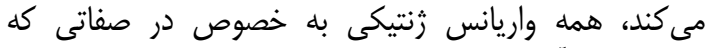

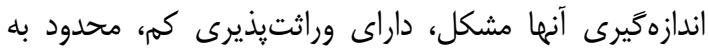

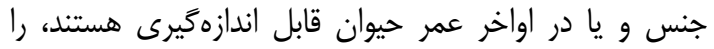

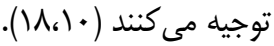

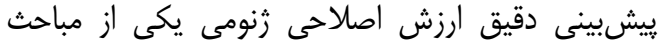

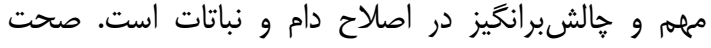

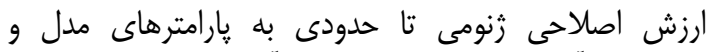

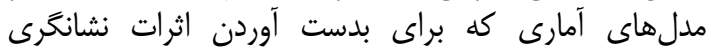

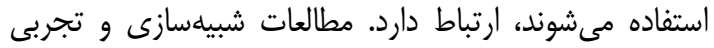

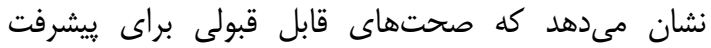

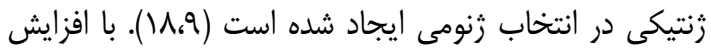




\section{مواد و روشها}

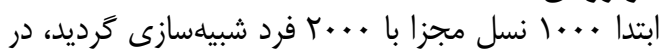

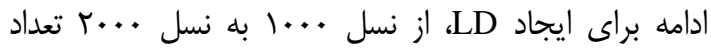

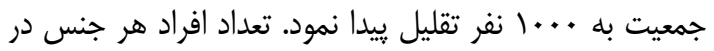

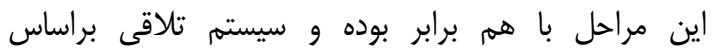

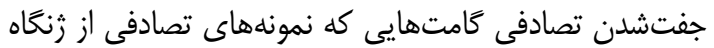

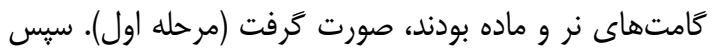

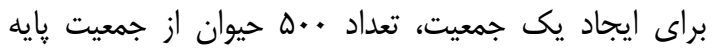

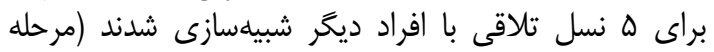

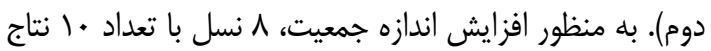
براى هر ماده شبيهسازى گرديد إنه (مرحله سوم). تمام حيوانات

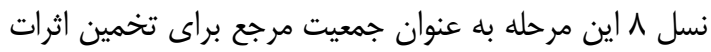

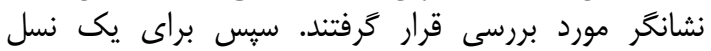

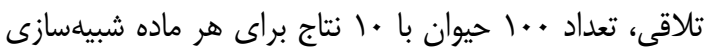

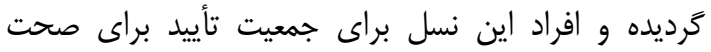

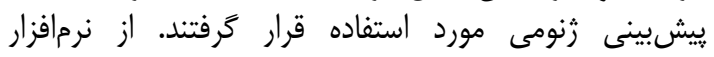

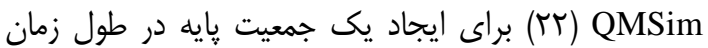

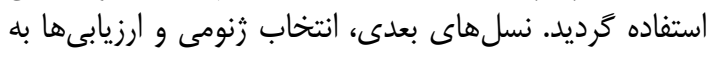

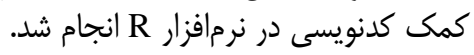

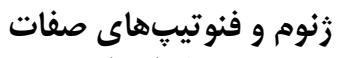

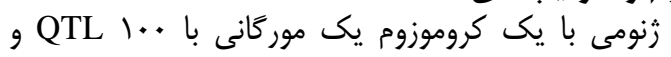

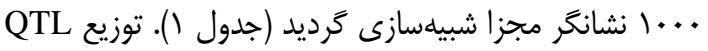

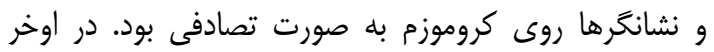

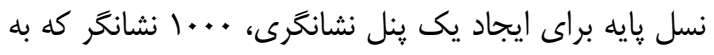

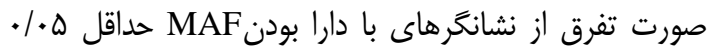

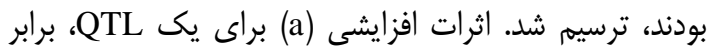

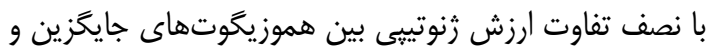

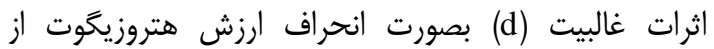

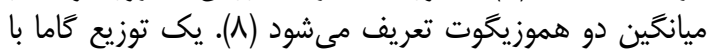

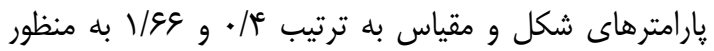

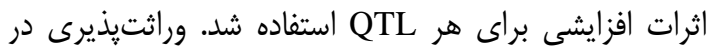

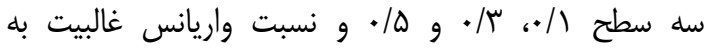

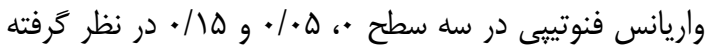

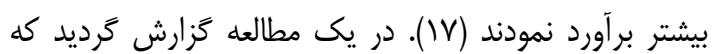

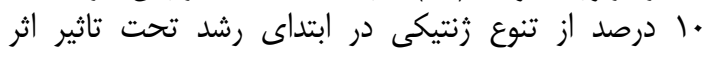

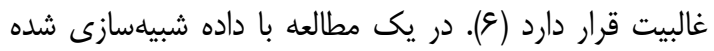

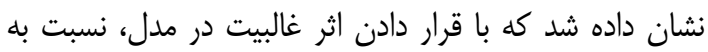

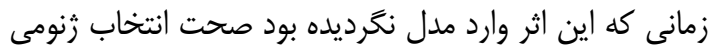

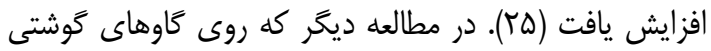

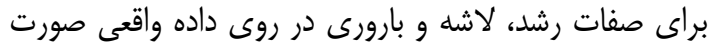

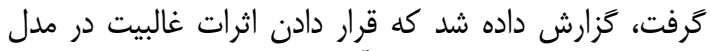

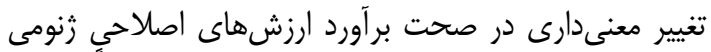

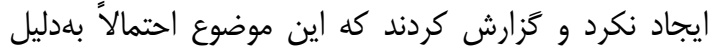

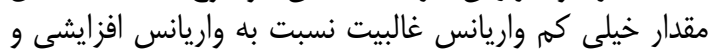

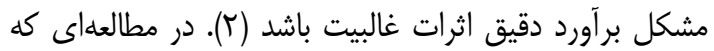

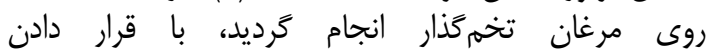

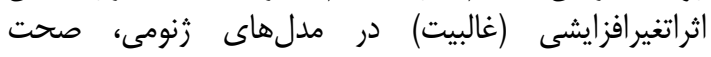
ييشبينى هاى زنومى را با سه روش

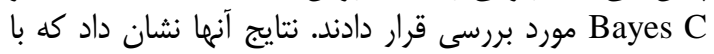

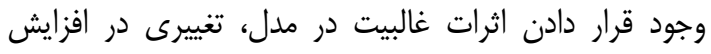

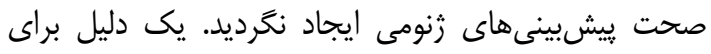

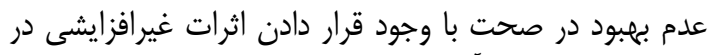

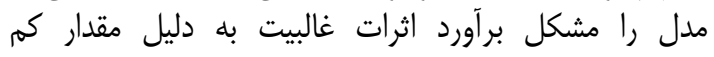

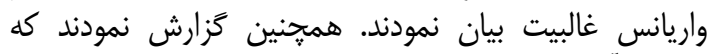

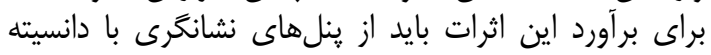

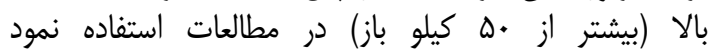

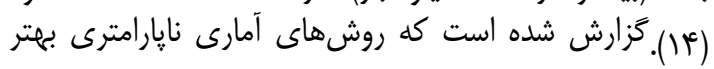

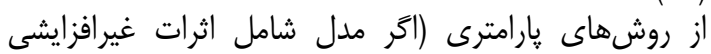

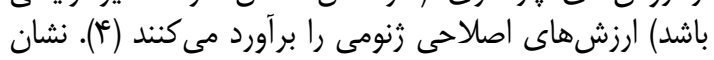

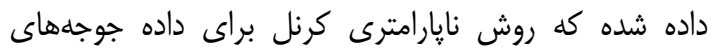

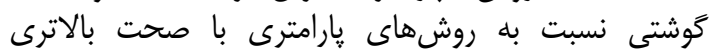

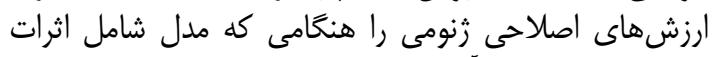

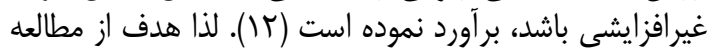

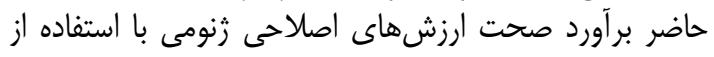

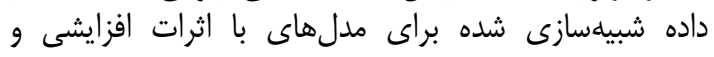

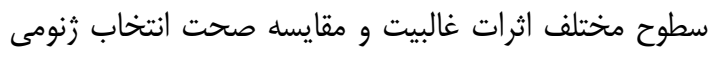

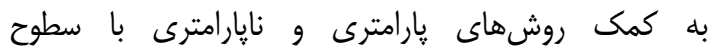
وراثتيذيرى متفاوت است.

Table 1. The structure of base populations and simulation parameters جدول ا- ساختار جمعيت يايه و یارامترهاى شبيهسازى شد

\begin{tabular}{|c|c|c|c|}
\hline مقدار & ( & مقدار & بِارامتر \\
\hline $1 \cdots$ & تعداد نشانخرها در طول ثنوم & نسل ( تا .... & ايجاد عدم تعادل بيوستخى \\
\hline $1 .$. & تعداد جايكاه صفات كمى & $r \cdot \Delta \Delta \in G+$. & جمعيت براى افزايش تعداد \\
\hline$r(\Delta x \mid)^{-r}$ & نرخ جهش & 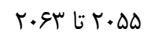 & جمعيت مرجع \\
\hline 1 & طول ثنوم (cM) & $r \cdot g r t r \cdot g r$ & جمعيت هدف \\
\hline كاما & اثرات افزايشى آللها براى QTL & $\begin{array}{c}1 \\
1 \ldots\end{array}$ & تعداد جمعيت مروزجع \\
\hline
\end{tabular}

اثرات نشانغر مىباشد. مدل آمارى براى تخمين اثرات نشانخر

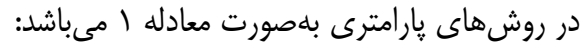
$y_{i}=\mu+\Sigma \mathrm{X}_{i j} a_{j}+\Sigma Z_{i j} d_{j}+e \quad$ (1) معادله

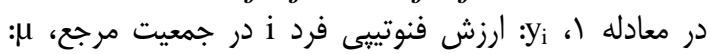

تخمين اثرات نشانكًر

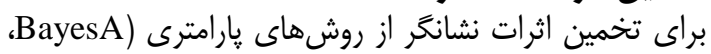

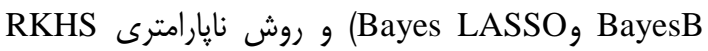
استفاده شد. تفاوت در روشهاى يارامترى در توزيع ييشين روارين 
صحت يِيشبينى زنومى

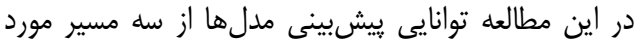

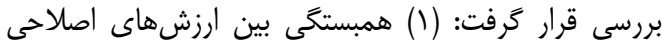

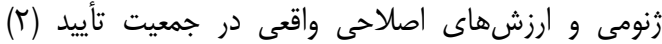

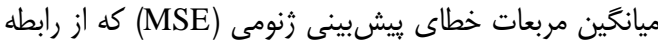
زير ييشبينى گرديد (1): $M S E=\frac{1}{N} \sum(G B V-T B V)^{2}$

معادله (f)

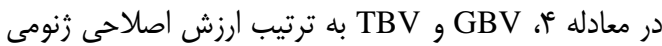

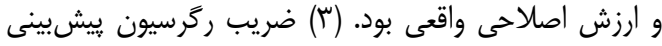

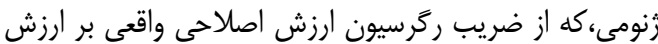

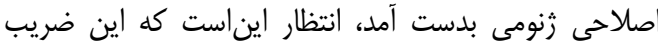

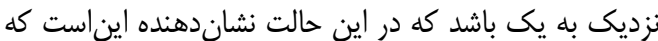
مقياس ارزش اصلاحى زنومى مشابه ارزش اصلاحى ترئ تخمينى مىباشد و اريب آن كمتر مى رباشد.

\section{نتايج و بحث}

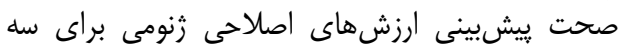

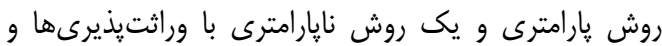

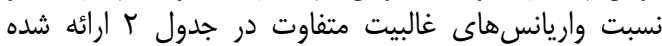

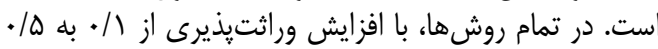

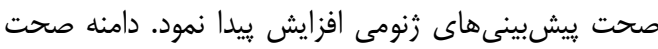

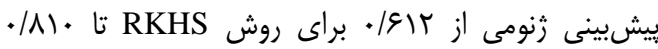

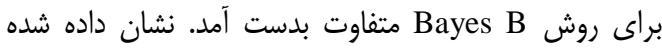

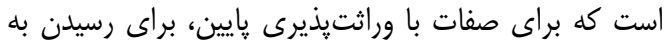

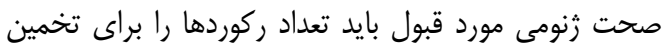

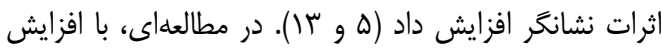

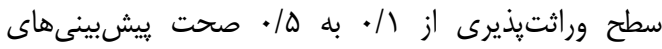

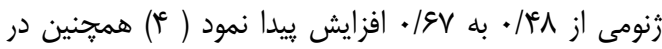

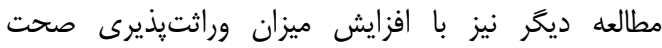

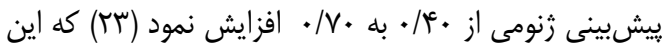

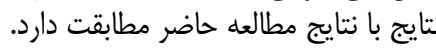

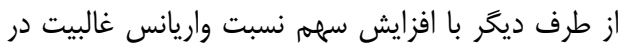

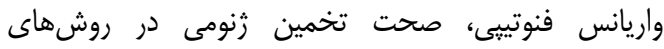

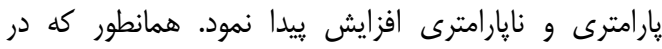

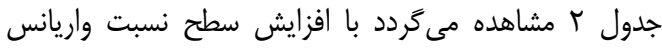

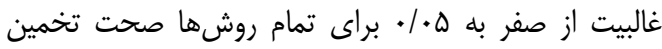

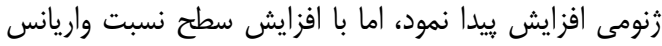

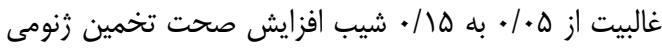

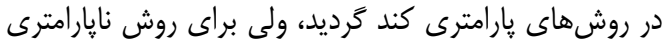

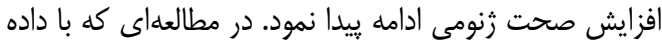

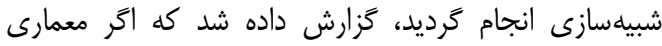

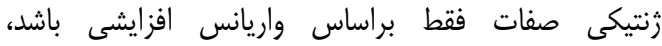

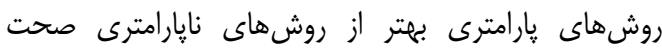

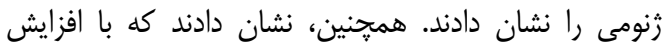

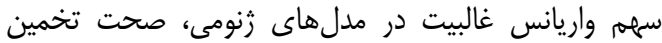

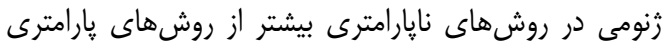

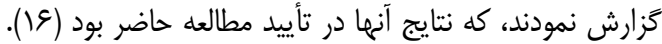

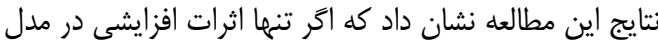

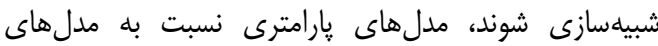

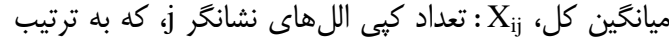

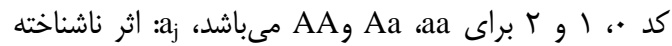

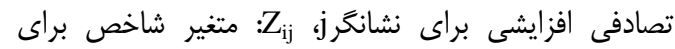

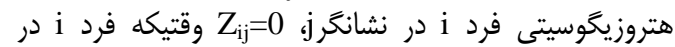

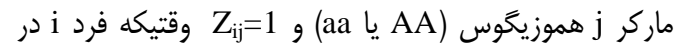

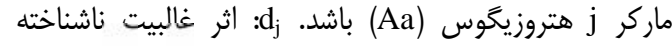

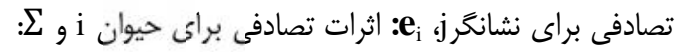

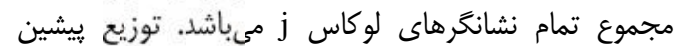
براى واريانس باقىمانده برابر با باس درجه آزادى و بارامتر مقياس (S

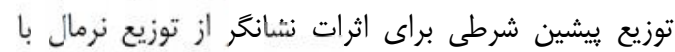

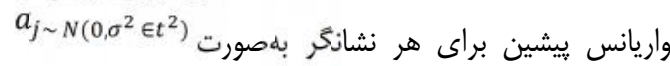

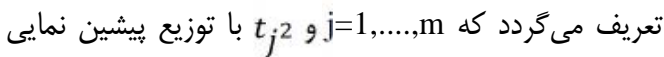
بلمورت

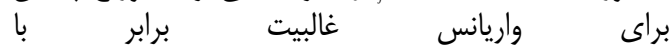
غوالئ $\sigma_{d}^{2} \sim \chi^{-2}\left(d f_{d}, S_{d}\right)$

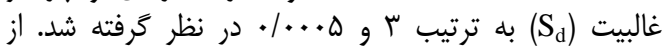

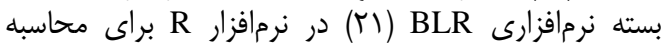

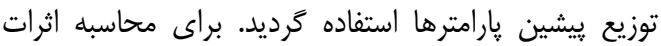

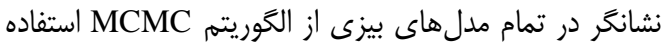

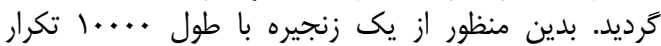

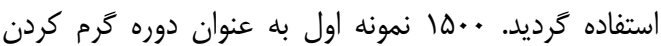
استفاده شد.

فضاى هيلبرت با هستهى باز آفرين

ReproducingKernel Hilbert Spaces (RKHS) اين روش به عنوان يكى روش نايارامترى براى برآيآورد

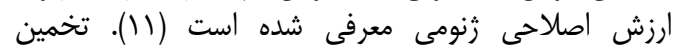

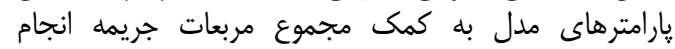

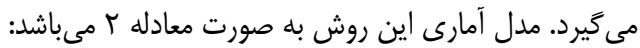

معادله (T)

$y_{i}=w_{i}^{\prime} \beta+z^{\prime} u+g\left(x_{i}\right)+e_{i}$,

در معادله r.,n,

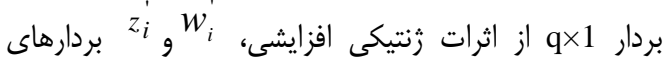

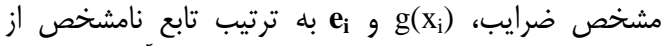

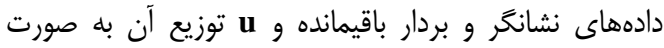

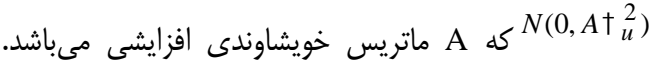
مجموع مربعات جريمه به صورت زير مىباشيد:

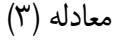

$S S(g(x), h)=\sum_{i=1}^{n}\left[y_{i}-w_{i}^{\prime} \beta-z_{i}^{\prime} u-g\left(x_{i}\right)\right]^{2}+h\|g(x)\|$,

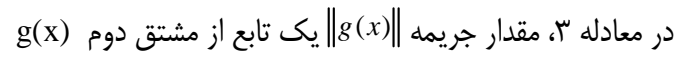

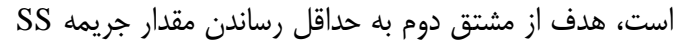

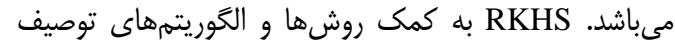

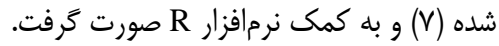


اصلاحى زنومى به كمك دادهاى شبيلهسازى شده كه به به

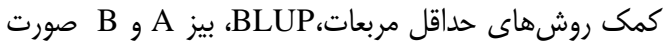

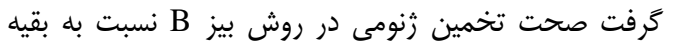

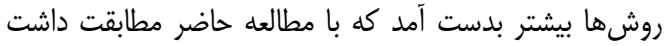

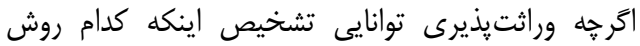

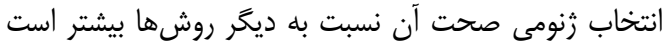

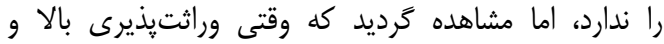

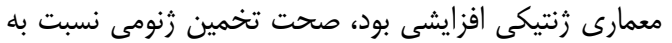

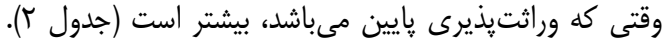

نايارامترى توان صحت ييشبينى زنومى آنها بيشتر بوده زيرا

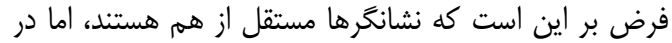

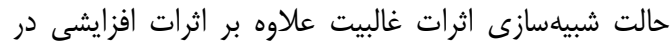

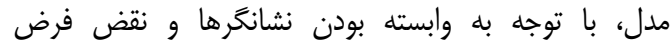

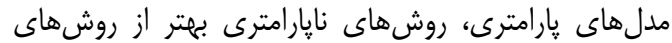

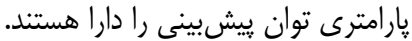

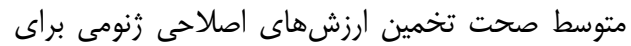

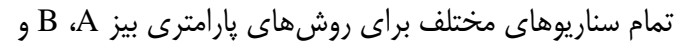

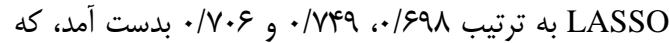

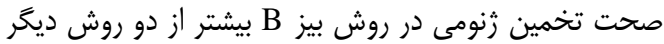

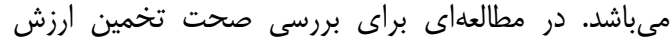

جدول r- صحت تخمين ارزش اصلاحى زنومى با روشهاى يارامترى و نا يارامترى براى وراثتيذيرىها و نسبتهاى واريانس غالبيت متفاوت Table 2. The accuracy of the prediction of genomic breeding value for parametric and non-parametric methods with

\begin{tabular}{|c|c|c|c|c|c|}
\hline RKHS & BayesLASSO & BayesB & BayesA & واريانس غالبيت & وراثتيذيرى \\
\hline . & - I\&QF &.$/ 899$ & 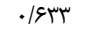 & - & \\
\hline.$/ 9 V \pi$ &.$/ 997$ & ( & $\cdot / 891$ & $\cdot 1 \cdot 0$ &.$/ 1$ \\
\hline .1990 &.$/ 9 \gamma$. & . $/$ VTr & $\cdot / 8 \times \Delta$ &.$/ 10$ & \\
\hline THK/. &.$/ 998$ & . MeT & $\cdot|91|$ & . & \\
\hline $.19 \mathrm{VF}^{\mathrm{C}}$ & . / M T & ./VGA & . /NTr & $.1 \cdot \Delta$ & r/. \\
\hline$\cdot / V \cdot r$ &.$/ 219$ & $\cdot / V K I$ & $\cdot / v \cdot 9$ &.$/ 10$ & \\
\hline.$/ 819$ & ./vq. & - IVAT & . NMr & . & \\
\hline . MIT & . MIT & $\cdot|\Lambda|$ & $\cdot / V^{*} \mid$ & $.1 \cdot \Delta$ & .10 \\
\hline.$/ V T I$ & $\cdot / V \cdot r$ & ./VפA & . &.$/ 1 \Delta$ & \\
\hline
\end{tabular}

براى روشهاى پارامترى ميانخين مربعات خطا افزايش يبيدا

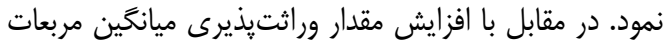

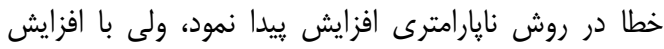

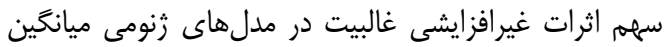

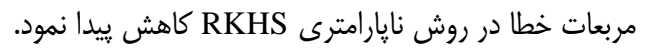

متوسط ميانگين مربعات خطا در روشهاى بيز A A LASSO

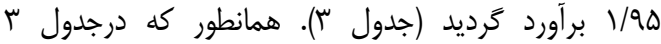

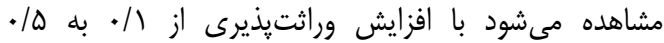

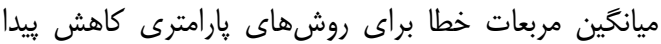
كرد، ولى با افزايش سهم اثرات غيرافئ مرايشى (غالبيت) در مدل

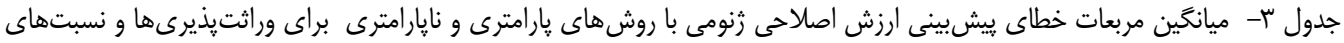
واريانس غالبيت متفاوت

Table 3. The mean squared prediction error of genomic breeding value for parametric and non parametric metl

\begin{tabular}{|c|c|c|c|c|c|}
\hline RKHS & BayesLASSO & BayesB & BayesA & واريانس غالبيت & وراثتيذيرى \\
\hline $1 / 91$ & 1/9T & $1 / \lambda r$ & 1/9 & . & \\
\hline $1 / \lambda r$ & $1 / \mathrm{VV}$ & $1 / 9 \Delta$ & I/VD & .1 .0 & .11 \\
\hline $1 / N r$ & $1 / A F$ & $1 / N r$ & I/A &.$/ 10$ & \\
\hline$r / l l$ & $1 / 19$ & $1 / V \Delta$ & $1 / \wedge \Delta$ & . & \\
\hline 1/9r & 1/94 & $1 / 9 \mathrm{~V}$ & $1 / \mathrm{V}$ & $.1 \cdot 0$ & $\cdot / \pi$ \\
\hline $1 / A V$ & $r / 11$ & $1 / N r$ & I/AT &.$/ 1 \Delta$ & \\
\hline$r / r)$ & $1 / v 8$ & $1 / \Delta 9$ & $1 / 99$ & - & \\
\hline $1 / 91$ & $1 / N T$ & 1/99 & I/VE & $\cdot 1 \cdot \Delta$ & $\cdot / \Delta$ \\
\hline $1 / M$ & I/Ar & $1 / N 1$ & $1 / \wedge 1$ &.$/ 10$ & \\
\hline
\end{tabular}

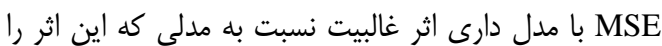

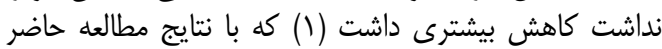

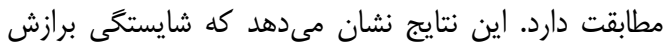

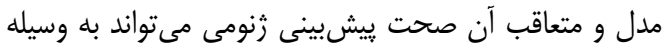

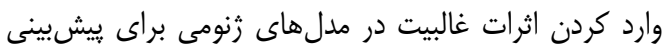

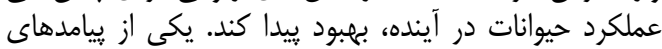

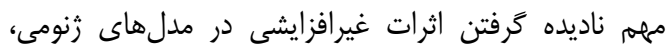

در يك مطالعه كه با داده شبيهسازى شده صورت گرفت،

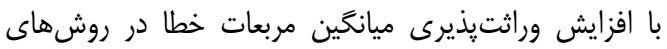

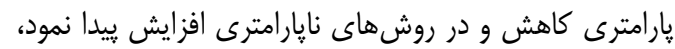

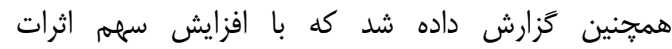

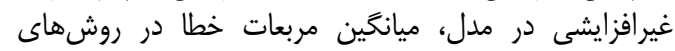

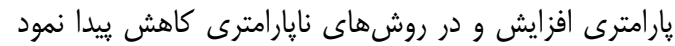

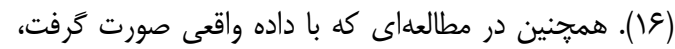


كردن اثرات غيرافزايشى غالبيت در مدل اريب اين روشها

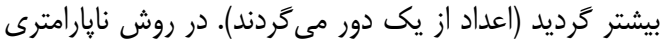

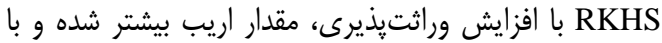

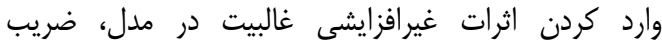

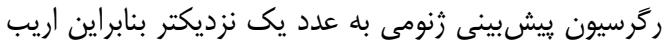

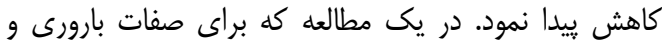

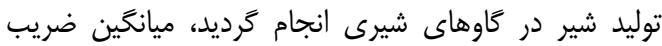

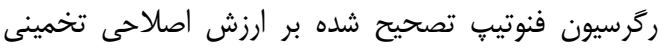

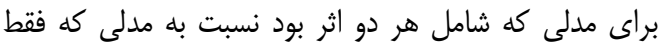

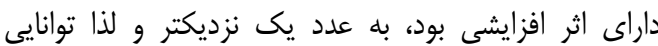

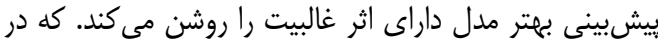

توافق با مطالعه حاضر بود (1).
اريبى ارزيابىهاى افراد و متعاقباً طبقهبندى نادرست افراد

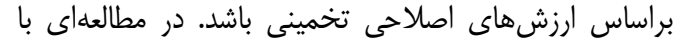

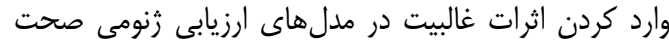

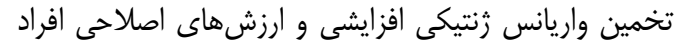

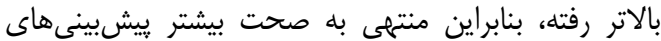

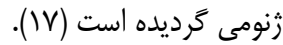

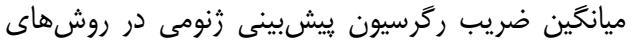

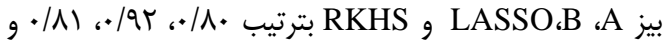

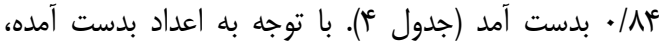

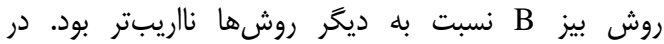

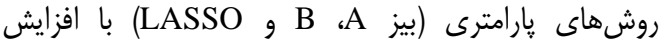

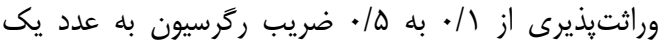

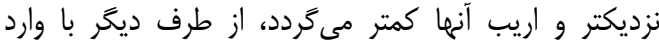

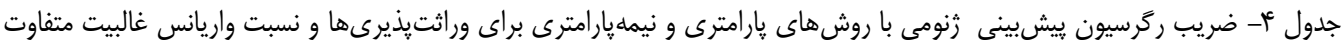
Table 4. The regression coefficient prediction of genomic breeding value for parametric and non-parametric methods

\begin{tabular}{|c|c|c|c|c|c|}
\hline RKHS & BayesLASSO & BayesB & BayesA & واريانس غالبيت & وراثتيذيرى \\
\hline$\cdot|\wedge|$ & $\cdot / A F$ &.$/ 94$ & •/А & - & \\
\hline . $/ \wedge \mu$ & ./vq & ./9T & $\cdot / v 9$ & $\cdot 1 \cdot 0$ &.$/ 1$ \\
\hline.$/ q$. & $\cdot / \mathrm{VA}$ &.$/ 199$ &.$/ \Lambda$ & .110 & \\
\hline$\cdot / v 9$ &.$/ M$ &.$/ 90$ & - / $\lambda F$ & - & \\
\hline - /Ar & ./Vq & ./9T & $\cdot / \Lambda$. & .1 .0 & . \\
\hline . /9r &.$/ \mathrm{r}$ &.$/ 9$. &.$/ V T$ &.$/ 1 Q$ & \\
\hline$\cdot / \mathrm{VA}$ &.$/ 95$ &.$/ 9 V$ & $\cdot / 1 / 9$ & . & \\
\hline . /Ar &.$/ M$ &.$/ 91$ & $\cdot / \Lambda$. & .1 .0 & .10 \\
\hline r/qu & r &.$/ 9$. &.$/ \mathrm{NF}^{\mathrm{C}}$ &.$/ 10$ & \\
\hline
\end{tabular}

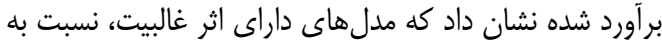

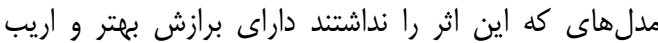

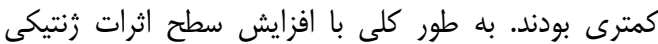

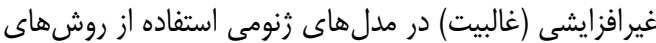

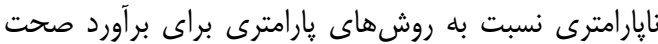
بيشبينى هاى زنومى توصيه مى كرداد
بطور كلى نتايج اين مطالعه نشان داد كه ا- با افزايش

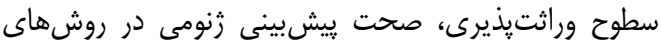

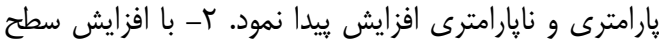

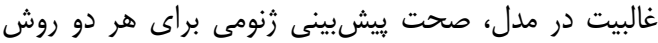

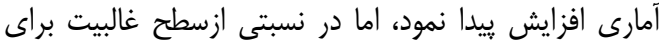

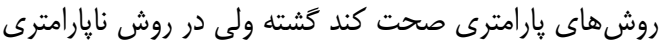

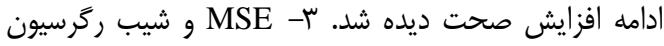


1. Aliloo, H., J.E. Pryce, O. Gonzalez-Recio, B.G. Cocks and B.J. Hayes. 2016. Accounting for dominance to improve genomic evaluations of dairy cows for fertility and milk production traits. Genetic Selection Evolution, 48: 8-11.

2. Bolormaa, S., J.E. Pryce1, Y. Zhang, A. Reverter, W. Barendse, B.J. Hayes and M.E. Goddard. 2015. Non-additive genetic variation in growth, carcass and fertility traits of beef cattle. Genetic Selection Evolution, 47(26): $12 \mathrm{pp}$.

3. Boysen, T.J., C. Heuer, J. Tetens, F. Reinhardt and G. Thaller. 2013. Novel use of derived genotype probabilities to discover significant dominance effects for milk production traits in dairy cattle. Genetics, 193: 431-42.

4. Calus, M.P.L. 2010. Genomic breeding value prediction: methods and procedures. Animal, 4(02): 157-164.

5. Calus, M.P.L., T.H.E. Meuwissen, A.P.W. De Roos and R.F. Veerkamp. 2008. Accuracy of genomic selection ussing different methods to define haplotypes. Genetics, 178: 553-561.

6. Carlborg, Ö., S. Kerje, K. Schütz,L. Jacobsson, P. Jensen and L. A. Andersson.2003.Global search reveals epistatic interaction between QTL for early growth in the chicken. Genome Research, 13: 413421.

7. de los Campos, G., D. Gianola, G.J.M. Rosa, K.A. Weigel and J. Crossa. 2010. Semi-parametric genomic-enabled prediction of genetic values using reproducing kernel Hilbert spaces methods. Genetic Research, 92: 295-308.

8. Falconer, D.S. and T.F.C. Mackay. 1996. Introduction to Quantitative Genetics. 4th ${ }^{\text {ed }}$. Harlow: Pearson Education Limited.

9. Gao, H., O.F. Christensen, P. Madsen, U.S. Nielsen, Y. Zhang, M.S. Lund and G. Su. 2012. Comparison on genomic predictions using three GBLUP methods and two single-step blending methods in the Nordic Holstein population. Genetic Selection Evolution, 44(8): 8 pp.

10. Gianola, D. and J.B. Van Kaam. 2008. Reproducing Kernel Hilbert Spaces Regression methods for genomic assisted prediction of quantitative traits. Genetics, 178: 2289-2303.

11. Goddard, M.E. and B.J. Hayes. 2007. Genomic selection. Journal Animal Breeding and Genetics, 124: 323-330.

12. González-Recio, O., D. Gianola, N. Long, K.A. Weigel, G.J.M. Rosa and S. Avendaño. 2008. Nonparametric methods for incorporating genomic information into genetic evaluations: An Application to Mortality in Broilers. Genetics, 178: 2305-2313.

13. Hayes, B.J., P.J. Bowman, A.J. Chamberlain and M.E. Goddard. 2009. Invited review: Genomic selection in dairv cattle: Progress and challenges. Journal Dairv Science. 92: 433-443.

14. Heidaritabar. M.. A. Wolc. J. Arango. J. Zeng. P. Settar. J.E. Fulton, N.P. Osullivan, J.W.M. Bastiaansen. R.L. Fernando, D.J. Garrick and J.C.M. Dekkers. 2016. Impact of fitting dominance and additive effects on accuracy of genomic prediction of breeding values in layers. Journal Animal Breeding and Genetics, 1-13.

15. Heslot, N., H.P. Yang, M.E. Sorrells and J.L. Jannink. 2012. Genomic selection in plant breeding: A Comparison of Models. Crop Science. 52: 146-160.

16. Howard. R.. A.L. Carriauirv and W.D. Beavis. 2014. Parametric and nonnarametric statistical methods for genomic selection of traits with additive and epistatic genetic architectures. G3, 4: 10271046.

17. Lee, S. H., J.H.J. Vander Werf, B.J. Hayes, M.E. Goddard and P.M. Visscher. 2008. Predicting unobserved phenotypes for complex traits from whole-genome SNP data. PLoS Genetic, 4: e1000231.

18. Meuwissen, T.H., B.J. Hayes and M.E. Goddard. 2001. Prediction of total genetic value using genome-wide dense marker mans. Genetics. 157: 1819-1829.

19. Neves, H., R. Carvalheiro and S.A. Queiroz. 2012. A comparison of statistical methods for genomic selection in a mice population. BMC Genetics, 13:100. $17 \mathrm{pp}$.

20. Ogutu, J.O., T. Schulz-Streeck and H.P. Piepho. 2012. Genomic selection using regularized linear regression models: ridge regression, lasso, elastic net and their extensions. BMC Proceedings. 6(Suppl 2): S10.

21. Perez, P., G. de los Campos, J. Crossa and D. Gianola. 2010. Genomic-enabled prediction based on molecular markers and pedigree using the Bayesian linear regression package in R. Plant Genome, 3: 106-16.

22. Sargolzaei, M. and F.S. Schenkel. 2009. QMSim: a large-scale genome simulator for livestock. Bioinformatics, 25: 680-1.

23. Saatchi, M., S.R. Miraei- Ashtiani, A. Nejati- Javaremi, M. Moradi-Shahrebabak and H. MehrabaniYeghaneh. 2010. The impact of information quantity and strength of relationship between training set and validation set on accuracy of genomic estimated breeding values. African Journal of Biotechnology, 9: 438-442.

24. Su, G., O.F. Christensen, T. Ostersen, M. Henryon and M.S. Lund. 2012. Estimating additive and non-additive genetic variances and predicting genetic merits using genome-wide dense single nucleotide polymorphism markers. PLoS One, 7(45): 293.

25. Wittenburg, D., N. Melzer and N. Reinsch. 2011. Including non-additive genetic effects in Bayesian methods for the prediction of genetic values based on genome-wide markers. BMC Genetics, 12-74. 


\title{
Genomic Selection Accuracy Parametric and Nonparametric Statistical Methods with Additive and Dominance Genetic Architectures
}

\begin{tabular}{c} 
Yahya Mohammadi ${ }^{\mathbf{1}}$ and Morteza Sattaei Mokhtari \\
\hline \hline 1- Assistant Professor, Department of Animal Science,University of Ilam, \\
(Corresponding Author: mohamadi_yahya @ yahoo.com) \\
2- Assistant Professor, Department of Animal Science, University of Jiroft \\
Received: February 13, $2017 \quad$ Accepted: Jun21, 2017 \\
\hline \hline
\end{tabular}

\begin{abstract}
In most genomic prediction studies only additive effects will be used in models for estimating genomic breeding values (GEBV). However, dominance genetic effects are an important source of variation for complex traits, considering them into account may improve the accuracy of GEBV. In the present study, performed applying simulated data, the effect of different heritability values $(0.1,0.3$ and 0.5$)$ and different values for the proportion of dominance variance to phenotypic variance $(0,0.05$ and 0.15$)$ on genomic selection accuracy in parametric (LASSO, A and B Bayes) and non-parametric (RKHS) statistical methods were studied. Correlations between the true and genomic breeding values, as a measure for the accuracy of genomic predictions under different scenarios were calculated using $\mathrm{R}$ software. The results of the present study revealed that, under all statistical methods as heritability values increased, the accuracy of genomic predictability increased. Also, as the value of dominance variance to phenotype variance increased genomic accuracy slant was slow under parametric methods but in the non-parametric method accuracy continued to increase. Under non parametric method the average mean square error was more reduced as the ratio of dominance variance to phenotype variance increased. Therefore, it may be concluded that under nonparametric method as the ratio of dominance variance to phenotype variance increased the accuracy of genomic predictions would be more increased than that of under parametric methods.
\end{abstract}

Keywords: Additive effect, Dominance effect, Genomic selection, Selection accuracy, Simulation 